\title{
Biomarker und Surrogat-Endpunkte: Garanten für eine schnellere Zulassung von neuen Arzneimitteln?
}

\author{
P. Kleist
}

\section{Zusammenfassung}

Biomarker nehmen eine zunehmende Bedeutung im Entwicklungsprozess neuer Arzneimittel ein. Da sie häufig jedoch nicht in der Lage sind, den tatsächlichen klinischen Wert und die Sicherheit eines Arzneimittels widerzuspiegeln, ist ihr Stellenwert im Rahmen der Marktzulassung gering. Für die Gesundheitsbehörden ist generell der auf Daten zu harten klinischen Endpunkten abgestützte Nachweis eines positiven Nutzen/RisikoVerhältnisses massgeblich. Nur in sehr wenigen Indikationsbereichen existieren validierte Surrogat-Endpunkte, die einen hohen Vorhersagewert für einen relevanten klinischen Endpunkt aufweisen - eine Zulassung allein auf der Basis eines Surrogat-Endpunktes stellt daher die Ausnahme dar. Dies trifft prinzipiell auch für Arzneimittel zur Therapie schwerwiegender, lebensbedrohender Erkrankungen zu, da die vorhandenen Verfahren zur Erteilung einer beschleunigten Zulassung auf Grundlage von vorläufigen Daten und ohne direkten Nachweis eines klinischen Nutzens nur in Einzelfällen von den Behörden angewendet werden. Nur wenn es gelingt, neue Biomarker als Surrogat-Endpunkte für harte klinische Endpunkte zu validieren, kann deren zukünftiger Stellenwert für die Zulassung neuer Arzneimittel steigen.

\section{Einleitung}

Sind die Anforderungen an die Zulassungsreife vielversprechender Substanzen zu hoch? Nimmt die Entwicklung bedeutsamer neuer Arzneimittel zu viel Zeit in Anspruch? Das 1992 in den USA implementierte beschleunigte Verfahren («accelerated approval») ermöglicht - im Gegensatz zum Standardverfahren - die Zulassung eines Arzneimittels zur Behandlung einer schweren, lebensbedrohlichen Erkrankung aufgrund vorläufiger Daten: Trotz eines noch fehlenden Nachweises des klinischen Nutzens, z.B. einer verlängerten Überlebenszeit, kann die Zulassung auf Basis der pharmakologischen Beeinflussung eines Surrogat-Endpunktes erteilt werden [1].

Kann ein beschleunigtes Verfahren wie das «accelerated approval» in den USA auf Basis von Surrogat-Endpunkten Modellcharakter für eine schnellere Marktreife neuer Arzneimittel mit dem Potential für einen signifikanten medizinischen Fortschritt aufweisen? Im folgenden Beitrag werden sowohl die Bedeutung von Biomarkern und Surrogat-Endpunkten für eine beschleunigte Entwicklung bzw. Zulassung von Arzneimitteln als auch die mit ihnen verbundenen Probleme und Gefahren im regulatorischen Prozess diskutiert.

\section{Definitionen}

Zunächst sollen die wichtigsten Begriffe erläutert werden, die nachfolgend in diesem Artikel verwendet werden $[2,3]$.

\section{Biologischer Marker (Biomarker)}

Ein objektiv messbarer und auswertbarer Indikator eines normalen biologischen Prozesses, eines pathologischen Prozesses oder einer pharmakologischen Antwort auf eine therapeutische Intervention. Klassische Beispiele für Biomarker, wie sie auch im klinisch-praktischen Alltag vorkommen, sind Laborwerte, die Knochendichte oder EKG-Parameter. Biomarkern kommt beispielsweise eine diagnostische oder prognostische Bedeutung $\mathrm{zu}$, sie haben jedoch an sich keinen Wert für den Patienten.

\section{Klinischer Endpunkt}

Eine Eigenschaft oder eine Variable, die das Befinden eines Patienten (z.B. Lebensqualitätsdaten oder Schmerzempfindung) oder seine Funktion beschreibt (z.B. Belastbarkeit). Bei schwer verlaufenden Erkrankungen besteht der Endpunkt meistens in der Frage nach der Verbesserung der Überlebenswahrscheinlichkeit. Ein klinischer Endpunkt beinhaltet somit einen direkten Wert für den Patienten. 
Tabelle 1

Validierte und seitens der Zulassungsbehörden akzeptierte Surrogat-Endpunkte; Referenzen im Text.

\begin{tabular}{lll}
$\begin{array}{l}\text { Therapie } \\
\text { Antihypertensiva }\end{array}$ & Surrogat-Endpunkt & Harte klinische Endpunkte \\
\hline Statine & Senkung des Serum LDL-Cholesterols & $\begin{array}{l}\text { Schlaganfälle, } \\
\text { Myokardinfarkte } \Downarrow\end{array}$ \\
\hline Orale Antidiabetika & Senkung des $\mathrm{HbA}_{1 c}$ & $\begin{array}{l}\text { Myokardinfarkte } \Downarrow, \\
\text { Überleben } \Uparrow\end{array}$ \\
\hline Bisphosphonate & Erhöhte Knochendichte & $\begin{array}{l}\text { Kardiovaskuläre } \\
\text { Komplikationen } \Downarrow\end{array}$ \\
\hline
\end{tabular}

Intermediärer (klinischer) Endpunkt

Die Messung einer klinisch relevanten Veränderung, die aber noch nicht den späten Krankheitsverlauf oder den endgültigen Krankheitsausgang widerspiegelt. Beispiele hierfür sind der Angina-pectoris-Schmerz bei koronarer Herzerkrankung oder die eingeschränkte Belastungstoleranz bei Herzinsuffizienz.

Harter (klinischer) Endpunkt

Er charakterisiert den endgültigen Ausgang einer Erkrankung, d.h. ihrer Heilung, das Auftreten schwerer Komplikationen oder ihren Tod.

\section{Surrogat-Endpunkt}

Hierbei handelt es sich um einen Biomarker oder einen intermediären Endpunkt, der einen hohen Vorhersagewert für einen harten klinischen Endpunkt aufweist.

\section{Die Bedeutung von Biomarkern und Surrogat-Endpunkten für die Arznei- mittelentwicklung und -zulassung}

Im Rahmen der Arzneimittelentwicklung nehmen Biomarker bereits heute einen hohen Stellenwert ein und tragen zu einer Verkürzung der Entwicklungszeit bei. In der präklinischen Phase erlauben sie die nicht-invasive Erfassung der Pharmakodynamik einer Substanz, werden für sogenannte Proof-of-Concept-Studien am Tier eingesetzt und können einen hohen prädiktiven Wert für toxikologische Eigenschaften haben. Humanpharmakologische Studien beruhen prinzipiell auf Biomarkern. Auch Proof-ofConcept-Studien am Menschen liegen häufig

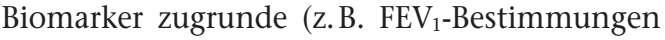
für neue Substanzen zur Asthmatherapie oder intragastrale $\mathrm{pH}$-Messungen bei Substanzen zur Behandlung peptischer Ulzera).

Dosis-Findungs-Studien werden häufig mit geeigneten pharmakodynamischen Endpunkten durchgeführt (z.B. Messung des Nüchtern- blutzuckers bei einem Antidiabetikum oder Auswahl eines geeigneten hämodynamischen Parameters bei einer Therapie der chronischen Herzinsuffizienz). Für die Diagnosestellung und Therapieverlaufsbetrachtungen stellen Biomarker eine wichtige Informationsquelle dar, z.B. die Bestimmung des PSA beim Prostatakarzinom oder des $\alpha$-Fetoproteins beim hepatozellulären Karzinom.

In klinischen Studien der späten Entwicklungsphase, in denen die Wirksamkeit und Sicherheit eines Arzneimittels an grossen Patientenkollektiven untersucht wird, spielen Biomarker bisher jedoch eine eher untergeordnete Rolle; nur in Einzelfällen dienen sie als Endpunkt für klinische Studien der Phasen IIB und III. In diesen besonderen Fällen finden Biomarker als Surrogat-Endpunkte Verwendung, d.h. sie sind in der Lage, einen harten klinischen Endpunkt zu ersetzen. Tabelle 1 spiegelt eine Auswahl von Substanzklassen wider, für deren Vertreter in der Vergangenheit eine konsistente Beziehung zwischen Beeinflussung eines Surrogat-Endpunktes und der Wirksamkeit in bezug auf einen harten klinischen Endpunkt aufgezeigt wurde [4-6]. Neue Substanzen dieser Klassen können von den Zulassungsbehörden allein auf der Basis der therapeutischen Beeinflussung des entsprechenden Surrogat-Endpunktes zugelassen werden. Da sich die Untersuchung der Wirksamkeit in bezug auf harte klinische Endpunkte erübrigt und somit keine Notwendigkeit für die Durchführung von Studien besteht, die sich über viele Jahre erstrecken würden, wird zwangsläufig die Entwicklungszeit solcher Arzneimittel verkürzt.

Bei einigen schwerwiegenden, lebensbedrohenden Erkrankungen wurde die Zulassung für neue Arzneimittel auf der Grundlage von Surrogat-Endpunkten erteilt, ohne dass zum Zulassungszeitpunkt eine Wirksamkeit auf klinische Endpunkte demonstriert wurde. Davon haben beispielsweise antiretrovirale Substanzen profitiert, deren Zulassung sich primär auf Erhöhungen der CD4+-T-Lymphozytenzahl und Reduktion der HIV-1 mRNA in Phase-I/II-Studien stützte [7]; die Annahme einer klinisch relevanten Wirkung beruhte allein auf der Tatsache, dass diesen Markern ein unabhängiger prognostischer Wert in bezug auf die Krankheitsprogression bei Patienten mit HIV-Infektion zukommt [8]. In vereinzelten Fällen wurden auch im Bereich der Onkologie neue, vielversprechende Arzneimittel auf Basis von Surrogat-Endpunkten zugelassen. Der BCR-ABL-Tyrosinkinase-Inhibitor Imatinib [9] erhielt die weltweite beschleunigte Zulassung aufgrund unerwartet hoher hämatologischer und zytogenetischer Ansprechraten. Der Nach- 
Tabelle 2

Beispiele für nicht-validierte Surrogat-Endpunkte; Referenzen im Text.

\begin{tabular}{|c|c|c|c|}
\hline Therapie & Indikation & Einfluss auf den Surrogat-Endpunkt & Einfluss auf klinische Endpunkte \\
\hline $\begin{array}{l}\text { Encainid, Flecainid, Moricizin } \\
\text { (Klasse-1C-Antiarrhythmika) }\end{array}$ & $\begin{array}{l}\text { Ventrikuläre Rhythmusstörungen } \\
\text { nach Myokardinfarkt }\end{array}$ & Reduktion ventrikulärer Extrasystolen & 2,5-fache Erhöhung der Mortalität \\
\hline $\begin{array}{l}\text { Milrinon } \\
\text { (Phosphodiesterasehemmer) }\end{array}$ & Herzinsuffizienz & $\begin{array}{l}\text { Verbesserte kardiale Funktion } \\
\text { (Herzindex } \Uparrow \text { ) }\end{array}$ & 28\%ige Erhöhung der Mortalität \\
\hline Hochdosis-Diuretikatherapie & Blutdruck $\Uparrow$ & Blutdrucksenkung & Erhöhung des KHK-Risikos \\
\hline Fibrate & Cholesterol $\Uparrow$ & Cholesterolsenkung & Gesamtmortalität $\Uparrow$ \\
\hline Dexfenfluramin & Übergewicht & Gewichtsverlust & Herzklappenschäden, pulmonale Hypertonie \\
\hline Natriumfluorid & Postmenopausale Osteoporose & Knochendichte $\Uparrow$ & Frakturrate $\Uparrow$ \\
\hline
\end{tabular}

weis der verlängerten Überlebenszeit ist durch andauernde klinische Studien noch zu erbringen.

Insgesamt zeigt sich, dass eine Zulassung auf der Grundlage von Surrogat-Endpunkten die Ausnahme und nicht die Regel ist. Auch im Bereich lebensbedrohlicher Erkrankungen wird das beschleunigte Zulassungsverfahren - abgestützt auf Surrogat-Endpunkte - nur in besonderen Einzelfällen angewendet. Insbesondere in der Onkologie fordern die Zulassungsbehörden nach wie vor den Nachweis der Wirksamkeit in bezug auf harte klinische Endpunkte, d.h. im Regelfall das Aufzeigen der verlängerten Überlebenszeit.

Warum spielen Surrogat-Endpunkte eine untergeordnete Rolle in der späten Entwicklungsphase eines Arzneimittels? Was sind die Gründe für die Zurückhaltung der Zulassungsbehörden, Marktzulassungen - auch mit vorläufigem Charakter - auf der Grundlage von SurrogatEndpunkten zu erteilen?

\section{Die grundsätzlichen Probleme mit Surrogat-Endpunkten}

Es existiert eine Vielzahl von Beispielen, die die mangelnde Akzeptanz von Surrogat-Endpunkten seitens der Behörden für die Arzneimittelzulassung erklären. Tabelle 2 zeigt Fälle auf, in denen die Übertragbarkeit von einem SurrogatEndpunkt (Biomarker oder intermediärer klinischer Endpunkt) auf einen harten klinischen Endpunkt nicht gegeben war. Die Beispiele werden nachfolgend systematisch erläutert.

\section{Surrogat-Endpunkte erfassen das Sicherheitsprofil einer Substanz nicht immer ausreichend}

Obwohl eine Korrelation zwischen dem Surrogat-Endpunkt und dem klinischen Endpunkt vorhanden sein kann, ist auf der Basis eines Sur-
rogat-Endpunktes keine abschliessende Nutzen/ Risiko-Abschätzung für ein Arzneimittel möglich. Surrogat-Endpunkte erfassen nicht das Sicherheitsprofil eines Arzneimittels (Abb. 1). Das hierfür eindrücklichste Beispiel lieferte die Cardiac Arrhythmia Suppression Trial (CAST-I/IIStudie) $[10,11]$, die zumindest im Bereich der Kardiologie zu einem Umdenken in bezug auf die Verwendung von Surrogatmarkern und die Notwendigkeit des Einsatzes einer antiarrhythmischen Therapie führte: Nahm man zuvor aufgrund historischer Daten an, dass die Reduktion ventrikulärer Extrasystolen grundsätzlich mit einer erhöhten Überlebenswahrscheinlichkeit in der Postinfarktphase verbunden ist, zeigte die CAST-Studie im Gegenteil sogar eine Erhöhung der Mortalität durch die in der Studie verwendeten Klasse-1C-Antiarrhythmika - wahrscheinlich bedingt durch ihre negativ-inotropen und eigenen proarrhythmogenen Eigenschaften.

In ähnlicher Weise trug die kontrollierte Untersuchung von Phosphodiesterasehemmern bei Herzinsuffizienz zur Ernüchterung bei: eine Verbesserung der hämodynamischen Situation durch Milrinon wurde durch die Auslösung von myokardischämiebedingten Arrhythmien antagonisiert, so dass als Nettoeffekt sogar eine gesteigerte Mortalität resultierte [12]. Im Gegensatz dazu ist z. B. die durch ACE-Hemmer induzierte Verbesserung der Hämodynamik mit einem klaren Überlebensvorteil verbunden.

Basierend auf den Daten einer kleinen Studie mit Dexfenfluramin [13] wurde die Schlussfolgerung gezogen, dass die Assoziation von Body Mass Index und Mortalität ein Surrogat für die erhöhte Überlebenswahrscheinlichkeit unter einer kontinuierlichen Therapie von Übergewichtigen mit dem Appetithemmer ist [14]. Die FDA erteilte eine Zulassung für Dexfenfluramin auf Basis dieser Surrogat-Endpunkt-Diskussion. Als sich jedoch herausstellte, dass das Risiko für schwere Nebenwirkungen höher war als 


\section{Abbildung 1}

Unzureichende Erfassung des klinischen Endpunktes durch einen Surrogat-Endpunkt, wenn andere Wirkmechanismen oder Langzeitnebenwirkungen von Bedeutung sind.

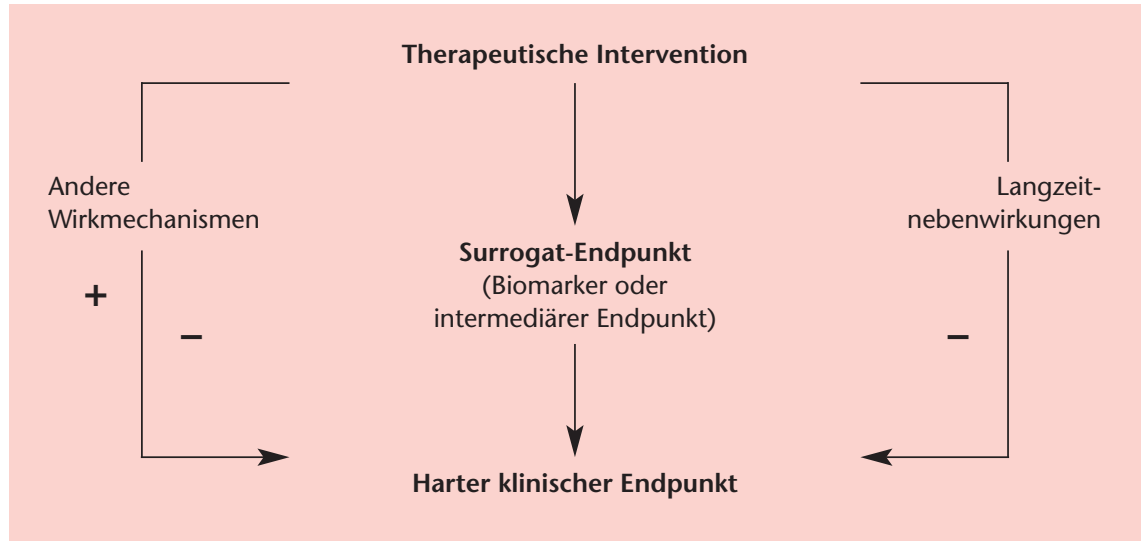

ursprünglich angenommen [15], musste die Substanz nach kurzer Zeit wieder vom Markt genommen werden.

\section{Surrogat-Endpunkte sind substanz- und dosisspezifisch}

Während für Statine eine hohe Korrelation zwischen Cholesterolreduktion und Mortalitätsreduktion gezeigt werden konnte [16, 17], sind Fibrate im Gegensatz dazu mit einer Erhöhung der Mortalität assoziiert [18]. Verschiedene Substanzen erhöhen die Knochendichte, aber eine Reduktion von Frakturen wurde im Rahmen kontrollierter Untersuchungen nur für wenige Substanzen gezeigt (z.B. für Bisphosphonate, Calcitonin oder Parathormon).

Die Beispiele verdeutlichen, dass ein SurrogatEndpunkt nicht an das allgemeine Wirkprinzip gekoppelt werden darf, sondern substanz-(klassen-)spezifisch betrachtet werden muss. Selbst unterschiedliche Dosen der gleichen Substanz können trotz gleichsinniger Effekte auf einen Surrogat-Endpunkt unterschiedliche klinische Effekte zur Folge haben. So sind niedrigdosierte Diuretika mit einem erniedrigten Risiko für kardiale Events verbunden, während hochdosierte Diuretika das kardiale Risiko erhöhen, trotz der ausgeprägteren Blutdrucksenkung [19] - wahrscheinlich bedingt durch die hypokaliämiebedingte Auslösung letaler Arrhythmien [20].

\section{Surrogat-Endpunkte erfassen unter Umständen nicht alle Wirkeigenschaften einer Substanz}

Obwohl sowohl Natriumfluorid als auch Bisphosphonate die Knochendichte erhöhen, steigert Natriumfluorid die Frakturrate [21], wäh- rend diese durch Bisphosphonate vermindert wird [22]. Diese Tatsache ist wahrscheinlich auf unterschiedliche Effekte auf den Knochenumbau zurückzuführen, die durch eine Messung der Knochendichte allein nicht zu erfassen sind (Abb. 1).

Bedingt durch den Umstand, dass nicht alle klinischen Effekte durch einen Surrogat-Endpunkt zu erfassen sind, kann das Wirkungsausmass einer Substanz oder einer Substanzklasse auch unterschätzt werden (Abb. 1). Neben der Cholesterolsenkung können theoretisch weitere potentielle Wirkmechanismen zur klinischen Wirksamkeit von Statinen beitragen, z. B. eine Plaque-Stabilisierung, Verbesserung der Endothelfunktion sowie antithrombotische und antiinflammatorische Eigenschaften [6], die durch die alleinige Cholesterolmessung nicht abgedeckt sind.

\section{Surrogat-Endpunkte sind indikations- spezifisch}

Das Auftreten einer Mikroalbuminurie besitzt beispielsweise einen relativ hohen Vorhersagewert für die kardiovaskuläre Mortalität und renale Morbidität bei Diabetikern; der diesbezügliche Vorhersagewert für nicht-diabetische Patientenpopulationen (z.B. für Hypertoniker) ist jedoch gering [23]. Ein weiteres Beispiel aus der Infektiologie soll ebenfalls die Indikationsspezifität von Surrogat-Endpunkten belegen: HIV-1 mRNA zeigt eine hohe Korrelation mit der Prognose bei HIV-Infektion; die Korrelation mit der Wirkung antiviraler Substanzen in bezug auf die Transmissionsrate von der Mutter auf das Neugeborene ist allerdings beschränkt [24].

Die vorherigen Beispiele verdeutlichen, warum die anfängliche Euphorie in den frühen 90er Jahren über den Gebrauch von Surrogaten, die auch die FDA 1992 mit der Einführung des Accelerated-approval-Verfahrens zu Änderungen ihrer Zulassungspraxis neuer Substanzen veranlasst hat [1], inzwischen von einer weitgehenden Ernüchterung abgelöst worden ist [25]. Die vor der Erteilung eines Zulassungsbescheides vorliegenden Daten grosser Interventionsstudien haben die Gesundheitsbehörden in einigen Fällen vor der Zulassung gefährlicher Substanzen bewahrt [26]; in einigen anderen Fällen mussten Produkte, die auf der Grundlage von SurrogatEndpunkten zugelassen wurden, wieder vom Markt genommen werden [15]. Welchen Stellenwert nehmen Biomarker und Surrogat-Endpunkte vor dem geschilderten Hintergrund bei den Zulassungsbehörden heutzutage ein? 


\section{Die gegenwärtige Sichtweise der Zulassungsbehörden}

Für die Marktzulassung einer neuen Substanz gilt grundsätzlich, dass sie neben dem Nachweis der Wirksamkeit auch den Nachweis der Sicherheit im Sinne eines positiven Nutzen/Risiko-Verhältnisses zu erbringen hat. Eine Substanz mit einer Wirkung auf einen Surrogat-Endpunkt, der nicht plausibel mit einem klinischen Endpunkt verbunden werden kann, ist nicht sicher - denn das Nutzen/Risiko-Verhältnis dieser Substanz kann nicht abgeschätzt werden [27]. Von den Behörden in Europa und den USA werden daher im Rahmen von Standardzulassungsverfahren nur validierte Surrogat-Endpunkte mit einem hohen prognostischen Wert für die Wirkung auf einen relevanten klinischen Endpunkt akzeptiert [28-30].

Welche Kriterien muss ein Surrogat-Endpunkt erfüllen, um valide zu sein? Ein validierter Endpunkt weist u.a. [2-4, 27, 31]

- eine hohe biologische Plausibilität auf;

- er spiegelt den Wirkungsmechanismus des Arzneimittels wider;

- er ist idealerweise spät im Krankheitsprozess angesiedelt;

- er beruht auf konsistenten epidemiologischen Daten;

- er ist durch Daten von verschiedenen Vertretern der gleichen therapeutischen Klasse, am besten durch Vertreter verschiedener therapeutischer Klassen validiert (FDA!);

- er hat eine hohe Sensitivität, Spezifität und Reliabilität (d.h. eine niedrige Variabilität und eine hohe Reproduzierbarkeit);

- er besitzt einen hohen Vorhersagewert in bezug auf den klinischen Endpunkt.

Dies erklärt, warum zurzeit nur wenige SurrogatEndpunkte von den Zulassungsbehörden als valide angesehen werden (Auswahl in Tab. 1). Diese betreffen Erkrankungen, bei denen die Untersuchung des harten klinischen Endpunktes (z. B. das Schlaganfallrisiko unter antihypertensiver Therapie) viele Jahre in Anspruch nehmen würde. In bezug auf neue, positiv-inotrop wirkende Substanzen ist in den USA aus dem gleichen Grund eine Zulassung auf Basis von intermediären klinischen Endpunkten prinzipiell möglich; es muss jedoch gezeigt werden, dass die neue Therapie nicht mit einem Anstieg der Sterblichkeit verbunden ist [27].

Hat ein neues Arzneimittel seine Wirksamkeit und Sicherheit auf Basis eines relevanten klinischen Endpunktes demonstriert, kann die Untersuchung von Biomarkern, d.h. von geeig- neten pharmakodynamischen Endpunkten, in zusätzlichen Bridging-Studien ausreichend sein, um die Zulassung auf andere Populationen zu erweitern. Ein solches Vorgehen ist in erster Linie in bezug auf andere ethnische Gruppen [32] und Kinder [33] anwendbar.

Handelt es sich um ernsthafte und lebensbedrohende Erkrankungen, so sind die Anforderungen für eine Zulassung auf Grundlage von Surrogat-Endpunkten weniger strikt als bei Standardzulassungsverfahren, da hier das medizinische Bedürfnis für neue Therapien grösser ist. Die FDA ermöglicht ein beschleunigtes Zulassungsverfahren (accelerated approval) für entsprechende Arzneimittel, wenn der Effekt auf einem Surrogat-Endpunkt beruht, der mit begründeter Wahrscheinlichkeit («reasonable likely») einen klinischen Nutzen vorherzusagen in der Lage ist $[34,35]$. Der klinische Nutzen ist durch PhaseIV-Studien nach der Zulassung zu belegen. Durch dieses Verfahren wurden die antiviralen Therapien zur Behandlung von HIV-Infektion/AIDS und einige onkologische Substanzen zugelassen (z.B. Capecitabin, Docetaxel, Irinotecan und Imatinib).

Im Rahmen des zentralisierten Zulassungsverfahrens in der EU existiert ebenfalls ein beschleunigtes Verfahren für Arzneimittel zur Therapie lebensbedrohender Erkrankungen [36]. Im Gegensatz zum amerikanischen Verfahren ist eine Zulassung auf Basis von Surrogat-Endpunkten nicht explizit erwähnt; die schnelle Zulassung von Imatinib zeigt jedoch, dass sie prinzipiell möglich ist. Das beschleunigte Verfahren wird in Europa jedoch nur in Ausnahmefällen gewährt: im Jahr 2001 erhielten nur zwei Arzneimittel auf diesem Weg ihre Zulassung [37]. In der Schweiz gibt es seit 1997 ein beschleunigtes Zulassungsverfahren [38], das bezüglich der Anwendungskriterien mit dem in der EU verglichen werden kann, im Gegensatz zum europäischen Verfahren aber weitaus häufiger genutzt wird. Eine Regelung bezüglich Surrogat-Endpunkten existiert wie in der EU nicht ausdrücklich; auch hier demonstrieren die Zulassung von Imatinib und die in den Erläuterungen zum Verfahren [38] genannten Beispiele (antivirale Substanzen) die prinzipielle Möglichkeit einer solchen Vorgehensweise durch die Schweizer Zulassungsbehörde.

Eine aktuelle Debatte betrifft den Zulassungszeitpunkt für neue Substanzen in der Onkologie. In den überwiegenden Fällen wurde die Zulassung nur aufgrund einer nachgewiesenen Erhöhung der Überlebenswahrscheinlichkeit und nicht auf Basis von Surrogat-Endpunkten erteilt. Denn obwohl der Vorhersagewert von Biomar- 
kern für den Verlauf maligner Erkrankungen zugenommen hat $[39,40]$, gibt es praktisch keine validierten Surrogat-Endpunkte in bezug auf die Überlebenswahrscheinlichkeit. Die Verfechter einer rascheren Zulassung von neuen Chemotherapeutika fordern dennoch von den Zulassungsbehörden, mehr Gewicht auf SurrogatEndpunkte, klinische Symptome und Lebensqualitätsdaten zu legen und die Nachweispflicht für eine lebensverlängernde Wirkung in die Postmarketingperiode zu verlagern [41, 42]. Die Zulassungsbehörden sind dem prinzipiellen Vorwurf ausgesetzt, mit zweierlei Mass zu messen, zumal die Zulassung antiviraler Substanzen oder einiger Enzymersatztherapien bei seltenen Stoffwechselerkrankungen ebenfalls ohne Überlebensdaten erfolgte [41]. Die Behörden fordern bei neuen, potentiell toxischen Therapien für maligne Erkrankungen jedoch weiterhin Daten zu klinischen Endpunkten, um auf dieser Basis die Sicherheit einer Substanz und deren Nutzen/ Risiko-Verhältnis zu bewerten. Ausnahmen von dieser Regel wird es nur bei äusserst vielversprechenden neuen Substanzen geben.

Damit ist auch die in der Einleitung gestellte Frage, ob das auf Surrogat-Endpunkten beruhende beschleunigte Zulassungsverfahren in den USA einen Modellcharakter für eine generell beschleunigte Zulassung von neuen Arzneimitteln haben kann, zumindest zum heutigen Zeitpunkt beantwortet. In bezug auf die Diskussion über den richtigen Zulassungszeitpunkt neuer Arzneimittel stellt sich für die Gesundheitsbehörden momentan viel eher die Frage, wie umfangreich die Datenlage einer neuen Substanz sein muss, um auf ihrer Basis eine solide Abschätzung der Arzneimittelsicherheit vornehmen zu können. Diese Frage ist jedoch nicht primär von SurrogatEndpunkten abhängig, sondern vom geforderten Ausmass der Wahrscheinlichkeit, mit der man Aussagen zur Sicherheit eines Arzneimittels vor der Zulassung machen kann [27].

\section{Ausblick}

Die Forschungen auf dem Gebiet der Genomik und Proteomik werden in Zukunft ein besseres Verständnis für die Pathogenese vieler Erkrankungen und die Identifikation neuer molekularer Targets von Arzneistoffen ermöglichen. In diesem Zusammenhang nimmt die Entwicklung neuer Biomarker zur Charakterisierung initialer Krankheitsprozesse und zur Erfassung von subklinischen Arzneimittelwirkungen eine herausragende Bedeutung ein. Die Hauptquellen für die Generierung neuer Biomarker stellen einerseits die Proteinbiochemie und andererseits nichtinvasive bildgebende Verfahren [43, 44] dar.

Neue Biomarker werden vor allem in den frühen Entwicklungsphasen von Arzneimitteln eine noch grössere Rolle als bisher spielen (Proofof-Concept; Dosiswirkungsstudien). Dies betrifft vor allem die Erfassung und Verlaufskontrolle von Arzneimittelwirkungen auf die Pathogenese von Erkrankungen, z. B. bei antiatherosklerotisch wirksamen Substanzen (Entzündungsproteine, Adhäsionsmoleküle, bildliche Darstellung der Gefässmorphologie und der Plaquebildung) oder in der Onkologie (Tumorwachstumsfaktoren, Faktoren der Angiogenese, Antigene, Kinasen) $[43,45,46]$. Eine auf einem Biomarker beruhende Arzneimittelentwicklung ist z. B. die von Imatinib zur Behandlung der CML. Die Substanz ist das Ergebnis eines gezielten Screenings für die optimale Hemmung der bei der Erkrankung aktivierten Tyrosin-Kinase [9].

Als weiteres Forschungsgebiet entwickelt sich momentan die Pharmakogenomik zur Standarddisziplin im Rahmen der präklinischen und klinischen Entwicklungsphasen eines Arzneimittels [47]. Durch Untersuchungen der Genexpression können neue Surrogatmarker für das Ansprechen und die Toxizität von Arzneimitteln gefunden werden [48]. Aufgrund von Erklärungen für die Variabilität von Arzneimittelwirkungen wird zukünftig ein gezieltes Screening von Patienten für klinische Studien ermöglicht [3, 49]. Ein Beispiel hierfür ist die Entwicklung von Trastuzumab, einem humanisierten Antikörper gegen den humanen epidermalen Wachstumsfaktor-Rezeptor-2 (HER2). In die klinischen Studien wurden nur Frauen aufgenommen, deren metastasierendes Mammakarzinom HER2 überexprimierte [50].

Für späte Entwicklungsphasen und die Marktzulassung wird aber nach wie vor der Nachweis des klinischen Nutzens eines neuen Arzneimittels von entscheidender Bedeutung sein. Ob neuen Biomarkern in Zukunft ein höherer Stellenwert für die Arzneimittelzulassung zukommt, hängt davon ab, ob ihre Validierung zu Surrogat-Endpunkten gelingt.

Im Rahmen von Interventionsstudien mit neuen Substanzen ist daher neben der Untersuchung harter klinischer Endpunkte auch eine umfangreiche Erfassung von Biomarkern notwendig, um Aussagen zu ihrer Korrelation mit dem klinischen Verlauf machen zu können. Ein gutes Beispiel für ein solches Vorgehen ist die Valsartan Heart Failure Trial (Val-HeFT), in der neben Ermittlung der Morbidität und Mortalität der Herzinsuffizienz bei allen Patienten die Effekte von Valsartan auf die linksventrikuläre 
Morphologie, die Ejektionsfraktion, verschiedene Neurohormone und die Lebensqualität und, im Rahmen von Substudien, auch auf Veränderungen im Holter-EKG, die Belastungstoleranz und das kardiale Remodelling abgeklärt wurden [51, 52]. Klinische Effekte lassen sich häufig eben nicht durch einen isolierten Biomarker abschätzen; darüber hinaus ist es wichtig zu beobachten, ob die Biomarker sich gleichgesinnt verhalten oder nicht. Beispielsweise liegt die Responderrate von Trastuzumab, trotz Selektion von Patientinnen, die HER2 überexprimieren, nur bei etwa 20\% [53]; dies bedeutet, dass andere Mechanismen eine zusätzliche Rolle spielen. Die zukünftige Erfassung der Wirksamkeit von Statinen wird daher aus dem gleichen Grund auf der Basis mehrerer Biomarker erfolgen, d.h. zumindest durch Messung des Cholesterols und des C-reaktiven Proteins [54].

\section{Schlussfolgerung}

Um zur Ausgangsfrage zurückzukehren: Sind Biomarker und Surrogat-Endpunkte Garanten für eine schnellere Zulassung von neuen Arzneimitteln? Zum heutigen Zeitpunkt generell nicht.

Sollte es in Zukunft gelingen, neue Biomarker im Rahmen von Interventionsstudien als Surrogat-Endpunkte für harte klinische Endpunkte zu validieren, könnte ihr genereller Stellenwert im Zulassungsprozess steigen. Die Menge und Vielfalt von neuen Biomarkern allein ändert deren Rolle für die Arzneimittelzulassung nicht. Die Abschätzung des Nutzen/Risiko-Verhältnisses auf Basis harter klinischer Endpunktdaten wird auch weiterhin die Grundlage für die Zulassung wirksamer und sicherer neuer Arzneimittel sein. 


\section{Literatur}

1 FDA/CDER. Fast track, priority review and accelerated approval. www.accessdata.fda.gov/ scripts/cder/onctools/Accel.cfm.

2 Biomarkers Definitions Working Group. Biomarkers and surrogate endpoints: Preferred definitions and conceptional framework. Clin Pharmacol Ther 2001;69:89-95

3 Lesko LJ, Atkinson Jr AJ. Use of biomarkers and surrogate endpoints in drug development and regulatory decision making: criteria, validation, strategies. Annu Rev Pharmacol Toxicol 2001; 41:347-66.

4 Colburn WA. Optimizing the use of biomarkers, surrogate endpoints, and clinical endpoints for more efficient drug development. J Clin Pharmacol 2000;40:1419-27.

5 Demol P, Weihrauch R. Surrogate endpoints. Their utility for evaluating therapeutic efficacy in clinical trials. Applied Clinical Trials 1998; October:46-54.

6 Psaty BM, Weiss NS, Furberg, CD, Koepsell TD, Siscovick DS, Rosendaal FR, et al. Surrogate end points, health outcomes, and the drug-approval process for the treatment of risk factors for cardiovascular disease. JAMA 1999;282:786-90.

7 Deyton L. Importance of surrogate markers in evaluation of antiviral therapy for HIV infection. JAMA 1996;276:159-60.

8 Marschner IC, Collier AC, Coombs RW, D'Aquila RT, DeGruttola V, Fischl MA, et al. Use of changes in plasma levels of human immunodeficiency virus type 1 RNA to assess the clinical benefit of antiretroviral therapy. J Infect Dis 1998;177:40-7.

9 Druker BJ, Talpaz M, Resta DJ, Peng B, Buchdunger E, Ford JM, et al. Efficacy and safety of a specific inhibitor of the BCR-ABL tyrosine kinase in chronic myeloid leukaemia. N Engl J Med 2001;344:1031-7.

10 Echt DS, Liebson PR, Mitchell B, Peters RW, Obias-Manno D, Barker AH, et al. Mortality and morbidity in patients receiving encainide, flecainide, or placebo. The Cardiac Arrhythmia Suppression Trial. N Engl J Med 1991;324:781-8.

11 CAST-II-Study - The Cardiac Arrhythmia Suppression Trial II-Investigators. Effect of the antiarrhythmic drug moricizine on survival after myocardial infarction. N Engl J Med 1992;327: 227-33.

12 Packer M, Carver JR, Rodeheffer RJ, Ivanhoe RJ, DiBianco R, Zeldis SM, et al. Effect of oral milrinone on mortality in severe chronic heart failure. The PROMISE Study Research Group. N Engl J Med 1991;325:1468-75.

13 Guy-Grand B, Apfelbaum M, Crepaldi G, Gries A, Levebvre P, Turner P. International trial of longterm dexfenfluramine in obesity. Lancet 1989; 2:1142-5.

14 Manson JE, Faich GA. Pharmacotherapy for obesity - do the benefits outweigh the risks (editorial)? N Engl J Med 1996;335:659-60.
15 McCann UD, Seiden LS, Rubin LJ, Ricaurte GA. Brain serotonin neurotoxicity and primary pulmonary hypertension from fenfluramine and dexfenfluramine. JAMA 1997;278:666-72.

16 Scandinavian Simvastatin Survival Study Group. Randomised trial of cholesterol lowering in 4444 patients with coronary heart disease: the Scandinavian Simvastatin Survival Study (4S). Lancet 1994;344:1383-9.

17 Shepherd J, Cobbe SM, Isles CG, Lorimer AR, MacFarlane PW, McKillop JH, Packard CJ. Prevention of coronary heart disease with pravastatin in men with hypercholesterolemia. West of Scotland Coronary Prevention Study Group. N Engl J Med 1995;333:1301-7.

18 Gould AL, Rossouw JE, Santanello NC, Heyse JF, Furberg CD. Cholesterol reduction yields clinical benefit. Circulation 1995;91:2274-82.

19 Multiple risk factor intervention trial. Risk factor changes and mortality results. Multiple Risk Factor Intervention Trial Research Group. JAMA $1982 ; 248: 1465-77$

20 Siscovick DS, Raghunathan TE, Psaty BM Koepsell TD, Wicklund KG, Lin X, et al. Diuretic therapy for hypertension and the risk of primary cardiac arrest. N Engl J Med 1994;330:1852-7.

21 Riggs BL, Hodgson SF, O'Fallon WM, Chao EY, Wahner HW, Muhs JM, et al. Effect of fluoride treatment on the fracture rate in postmenopausal women with osteoporosis. N Engl J Med 1990; 322:802-9.

22 Pols HA, Felsenberg D, Hanley DA, Stepan J, Munoz-Torres M, Wilkin TJ, et al. Multinational, placebo-controlled, randomised trial of the effects of alendronate on bone density and fracture risk in postmenopausal women with low bone mass: results of the FOSIT study. Fosamax International Trial Study Group. Osteopor Int 1999;9:461-8.

23 Epstein M, Parving H-H, Ruilope LM. Surrogate endpoints and renal protection: focus on microalbuminuria. Blood Pressure 1997;6(suppl 2):52-7.

24 Sperling RS, Shapiro DE, Coombs RW, Todd JA, Herman SA, McSherry GD, et al. Maternal viral load, zidovudine treatment, and the risk of transmission of human immunodeficiency virus type 1 from mother to infant. Pediatric AIDS Clinical Trials Group Protocol 076 Study Group. N Engl J Med 1996;335:1621-9.

25 Fleming TR, DeMets DL. Surrogate endpoints in clinical trials: are we being misled? Ann Intern Med 1996;125:605-13.

26 Packer M, Narahara KA, Elkayam U, Sullivan JM, Pearle DL, Massie BM, Creager MA. Double-blind, placebo-controlled study of the efficacy of flosequinan in patients with chronic heart failure. Principal Investigators of the REFLECT Study. J Am Coll Cardiol 1993;22:65-72.

27 Temple R. Are surrogate markers adequate to assess cardiovascular disease drugs? JAMA 1999 282:790-5.

28 ICH Topic E8. Note for guidance on general considerations for clinical trials. CPMP/ICH/291/95. July 1997. www.emea.eu.int. 
29 ICH Topic E9. Note for guidance on statistical principles for clinical trials. CPMP/ICH/363/96. February 1998. www.emea.eu.int.

30 FDA/CDER/CBER. Guidance for industry. Providing clinical evidence of effectiveness for human drug and biological products. May 1998. www.fda.gov/cder/guidances.

31 Schellhammer P, Cockett A, Boccon-Gibod L, Gospodarowicz M, Krongrad A, Thompson IM et al. Assessment of endpoints for clinical trials for localized prostate cancer. Urology 1997;49 (suppl 4A):27-38.

32 ICH Topic E5. Note for guidance on ethnic factors in the acceptability of foreign clinical data. CPMP/ICH/289/95. February 1998. www.emea.eu.int.

33 ICH Topic E11. Note for guidance on clinical investigation of medicinal products in the paediatric population. CPMP/ICH/2711/99. July 2000. www.emea.eu.int.

34 Food and Drug Administration Modernization Act (FDAMA). §112 (1997).

35 New drug, antibiotic, and biological drug product regulations. Accelerated approval. Final rule. 57 Federal Register 58942-58960 (1992).

36 The European Agency for the Evaluation of Medicinal Products. Accelerated evaluation of products indicated for serious diseases (life threatening or heavily disabling diseases). CPMP/495/96 rev.1. September 2001. www.emea.eu.int.

37 The European Agency for the Evaluation of Medicinal Products. $7^{\text {th }}$ Annual report. Adopted December 2001. www.emea.eu.int.

38 Swissmedic Erläuterungen. Das beschleunigte Zulassungsverfahren (BZV). www.swissmedic.ch.

39 Lindblom A. Tumour markers in malignancies. Br Med J 2000;320:424-7.

40 Watanabe T, Wu TT, Catalano PJ, Ueki T, Satriano R, Haller DG, et al. Molecular predictors of survival after adjuvant chemotherapy for colon cancer. N Engl J Med 2001;344:1196-207.

41 Koopmans PP. Clinical endpoints in trials for cancer: a time for a rethink? Br Med J 2002 324:1389-91.

42 Kessler DA, Feiden KL. Faster evaluation of vital drugs. Sci Am 1995;272:48-54.
43 Feinstein SB, Voci P, Pizzuto F. Noninvasive surrogate markers of atherosclerosis. Am J Cardiol 2002;89(suppl):31C-44C.

44 Nissen S. Coronary angiography and intravascular ultrasound. Am J Cardiol 2001;87(suppl): 15A-20A.

45 Stein E. Laboratory surrogates for antiatherosclerotic drug development. Am J Cardiol 2001; 87(suppl):21A-26A.

46 Sharma RA, Harris AL, Dalgleish AG, Steward WP. O'Byrne KJ. Angiogenesis as a biomarker and target in cancer chemoprevention. Lancet Oncology 2001;2:726-32.

47 Collins F, McKusick V. Implications of the human genome project for medical science. JAMA 2001; 285:540-4.

48 Rininger JA, DiPippo VA, Gould-Rothberg BE. Differential gene expression technologies for identifying surrogate markers of drug efficacy and safety. Drug Discov Today 2000;5:560-8.

49 Haberkorn U, Altmann A, Eisenhut M. Functional genomics and proteomics - the role of nuclear medicine. Eur J Nucl Med 2002;29:115-32.

50 Shak S, Herceptin Multinational Investigators Study Group. Overview of the trastuzumab (Herceptin) anti-HER2 monoclonal antibody clinical program in HER2-overexpressing metastatic breast cancer. Semin Oncol 1999; (suppl 12):71-7.

51 Cohn JN, Tognoni G. A randomized trial of the angiotensin-receptor blocker valsartan in chronic heart failure. N Engl J Med 2001;345:1667-75.

52 Cohn JN, Tognoni G, Glazer RD, Spormann D, Hester A. Rationale and design of the Valsartan Heart Failure Trial: a large multinational trial to assess the effects of valsartan, an angiotensinreceptor blocker, on morbidity and mortality in chronic congestive heart failure. J Card Fail 1999;5:155-60.

53 Ravdin PM. Should HER2 status be routinely measured for all breast cancer patients? Semin Oncol 1999;26(suppl 12):117-23.

54 Ridker PM, Rifai N, Pfeffer MA, Sacks F, Braunwald E. Long-term effects of pravastatin on plasma concentration of C-reactive protein. The Cholesterol and Recurrent Events (CARE) Investigators. Circulation 1999;100:230-5 